\title{
Structural studies of a fungal polyphenol oxidase with application to bioremediation of contaminated water $^{\dagger}$
}

\author{
Alexandros Valmas ${ }^{1, *}$, Grigorios Dedes ${ }^{2}$ and Maria Dimarogona ${ }^{3, *}$ \\ 1 Department of Biology, University of Patras, Patras, Greece, valmas@upatras.gr \\ 2 Biotechnology Laboratory, School of Chemical Engineering, National Technical University of Athens, \\ Athens, Greece; gdedes@chemeng.ntua.gr \\ 3 Department of Chemical Engineering, University of Patras, Patras, Greece; mdimarog@chemeng.upatras.gr \\ * Correspondence: (A.V.) valmas@upatras.gr; (M.D.) mdimarog@chemeng.upatras.gr \\ + Presented at the 1st International Electronic Conference on Microbiology, 02-30 November 2020; Available \\ online: https://ecm2020.sciforum.net/ \\ Published: 02 November 2020
}

\begin{abstract}
Polyphenol oxidases (PPOs) are a group of $\mathrm{Cu}$-containing enzymes exhibiting two activities, catechol oxidase and tyrosinase. Their precise mechanism of action and the structural elements that determine the distinction between the two activities are yet to be fully understood. In nature, PPOs catalyse the oxidation of several phenols to o-quinones, considerably affecting the colour and nutritional properties of numerous agricultural products. On the other hand, PPOs have been widely employed as biocatalysts in food, pharmaceutical, and cosmetic industries. TtPPO is a PPO from the thermophilic fungus Thermothelomyces thermophila (TtPPO), capable of degrading of chlorophenols (CPs), contagious by-products of various pesticides. The present work aims to clarify the structural determinants of TtPPO function, by performing protein-ligand docking experiments via YASARA software. The docking results are compared with biochemical data, and the role of specific amino acids in TtPPO function is investigated. The identification of the amino acids involved in binding of the different substrates to the active site of the enzyme would allow the structure-based design of a more efficient biocatalyst for wastewater treatment.
\end{abstract}

Keywords: polyphenol oxidase; tyrosinase; phenol derivative; docking; structure-function relations

\section{Introduction}

Polyphenol oxidases (PPOs) are a group of metalloenzymes containing a type-III copper centre and are distributed among bacteria, fungi, archaea, plants, insects and animals [1]-[5]. Type III copper center consists of two copper ions $(\mathrm{CuA}$ and $\mathrm{CuB})$ coordinated by three histidine residues each [6]. PPOs exhibit two different enzymatic activities: a. Tyrosinase (L-tyrosine, L-DOPA:oxygen oxidoreductase; EC 1.14.18.1), which catalyzes the ortho-hydroxylation of monophenols and the subsequent two electron oxidation of the o-diphenols to the corresponding o-quinones, and $\mathrm{b}$. Catechol oxidase (1,2-benzene-diol:oxygen oxidoreductase; EC 1.10.3.1), which only catalyzes the latter diphenolase reaction [7]. The quinones produced as a result of PPO activity lead to the production of complex polymers known as melanins, which cause enzymatic browning in fruits and vegetables, considerably reducing their market value. [8]. One of the most important although least studied application of PPOs is as sensitive detectors of phenol derivatives in polluted waters [9] as well as efficient tools for the biodegradation of these substances [10].

In spite of various crystallographic and molecular docking studies, the structural determinants that affect the distinction between the two enzymatic activities and the substrate specificity exhibited by PPOs are still not fully understood. For instance, molecular studies on two walnut (Juglans regia) 
tyrosinase isoenzymes ( $J r P P O 1$ and $J r P P O 2)$ showed that the activity towards monophenolic substrates was dependent on the occurrence of an asparagine residue located after one of the $\mathrm{CuB}$ coordinating histidines [11]. However, there are many PPOs that do not have an Asn residue in this position but still display monophenolase activity [7].

The present work is focused on TtPPO, a PPO from the thermophilic fungus Thermothelomyces thermophila that is capable of degrading chlorophenols (CPs), contagious by-products of pesticides [12]. Various TtPPO mutants, designed based on a structural model and according to existing literature, were previously produced and biochemically characterized. In order to shed light on the structure-function relations of TtPPO, we determined the tertiary structure of TtPPO mutant (G292N) by X-ray crystallography (PDB code 6Z1S), however, subsequent efforts to determine TtPPO structure in complex with various substrate analogues have not been yet successful. In the present work, we used YASARA software to dock 3,4-Dihydroxy-L-phenylalanine (L-DOPA) on native $T t P P O$ and 2 mutants that exhibited substantial differentiations in their catalytic efficiency. Based on the docking results, we discuss the implication of specific aminoacids on substrate specificity of TtPPO.

\section{Methods}

Enzyme-substrate docking experiments were performed using YASARA software [13]. The crystal structure of TtPPO (mutant G292N, PDB entry 6Z1S) was prepared for molecular docking as a two-step process. Initially, the pdb file was introduced to $Y A S A R A$ and water molecules, ligands, ions as well as oligosaccharides from glycosylation site were excluded from the structure. Next, all missing hydrogen atoms were added and residues having several conformations were identified (only one of the multiple conformations was kept). The structure of L-DOPA (in pdb format) was obtained from Ligand Expo [14], and formatted into YASARA-object files (*.yob) for later use. YASARA offers two docking methods, either AutoDock LGA [15-16] or VINA [17]. Binding poses were searched in a simulation cell, extended at $11 \AA$ around the two copper ions of the active site. AutoDock VINA was selected as docking method while the exhaustiveness was set to 25 and the same number of poses were calculated for each target and substrate. Poses that significantly deviated from the expected binding pose were "flagged as 'unreasonable' poses. All visualizations were created using PyMOL 2.3.2 (The PyMOL Molecular Graphics System, Version 2.3.2, Schrödinger, LLC).

\section{Results and Discussion}

The original purpose of the present work was to analyze the structural determinants that affected the specificity of TtPPO, a recently purified and characterized oxidase [12]. Interestingly, $T t P P O$ had the ability to degrade various chlorophenols, rendering it a biocatalyst of biotechnological interest. In order to be able to improve TtPPO efficiency as a bioremediation agent, via protein engineering, a detailed knowledge of substrate binding in its active site is a prerequisite. We thus performed docking experiments, using various phenolic substances, including chlorophenols. However, all docking attempts using chorophenols resulted in their positioning outside the expected substrate binding pocket. L-DOPA, on the contrary, seemed to be located in a catalytically favorable position. This finding, in combination with the fact that mutation of specific aminoacids affected substantially TtPPO efficiency against this particular substrate led us to use these docking results in order to identify aminoacids in TtPPO active site, actively implicated in its oxidative efficiency.

More specifically, kinetic studies of TtPPO and its mutants, using a variety of substrates, showed that the double mutation G292N/L306A resulted in a variant with significantly increased activity on L-DOPA (data not shown). The latter was therefore chosen as docking target, and the relevant results are presented in Table 1.

Table 1. Molecular docking results of L-DOPA on native TtPPO and mutants G292N, 


\begin{tabular}{ccc}
\hline & $\begin{array}{c}\text { Best Bind.energy } \\
{[\mathbf{k c a l} / \mathbf{m o l}]}\end{array}$ & Dissoc. constant $[\boldsymbol{\mu M}]$ \\
\hline Native & 4.937 & 240.508 \\
\hline G292N & 5.097 & 183.590 \\
\hline G292N/L306A & 5.614 & 76.715 \\
\hline
\end{tabular}

The predicted binding poses are quite close to the crystallographically determined location of ligands in PPO active site, with the bound ligand being positioned between the two active site copper ions [1]. As shown in Figure 1A, L-DOPA is stabilized in native TtPPO by forming hydrogen bonds with residues Ser309, His291 and Tyr119. It also seems to form a $\pi-\pi$ stacking interaction with His295. When glycine 292 is mutated to asparagine (Fig. 1B), the aliphatic part of the ligand is shifted towards the opposite direction, forming additional hydrogen bonds with the backbone carbonyls of G304, L306 and N292, as well as with the side chain hydroxyl of Tyr296. In addition, the molecule shifts closer to the active site copper ions. When additional mutation L306A is introduced (Fig. 1C), resulting in a less stereochemically congested active site, the aliphatic side chains shifts back to its original position. In this case, L-DOPA seems to be stabilized in the active site by a higher number of hydrogen bonds, when compared to the native enzyme, involving amino acids Ser309, Tyr119, Asn292 and Tyr296.

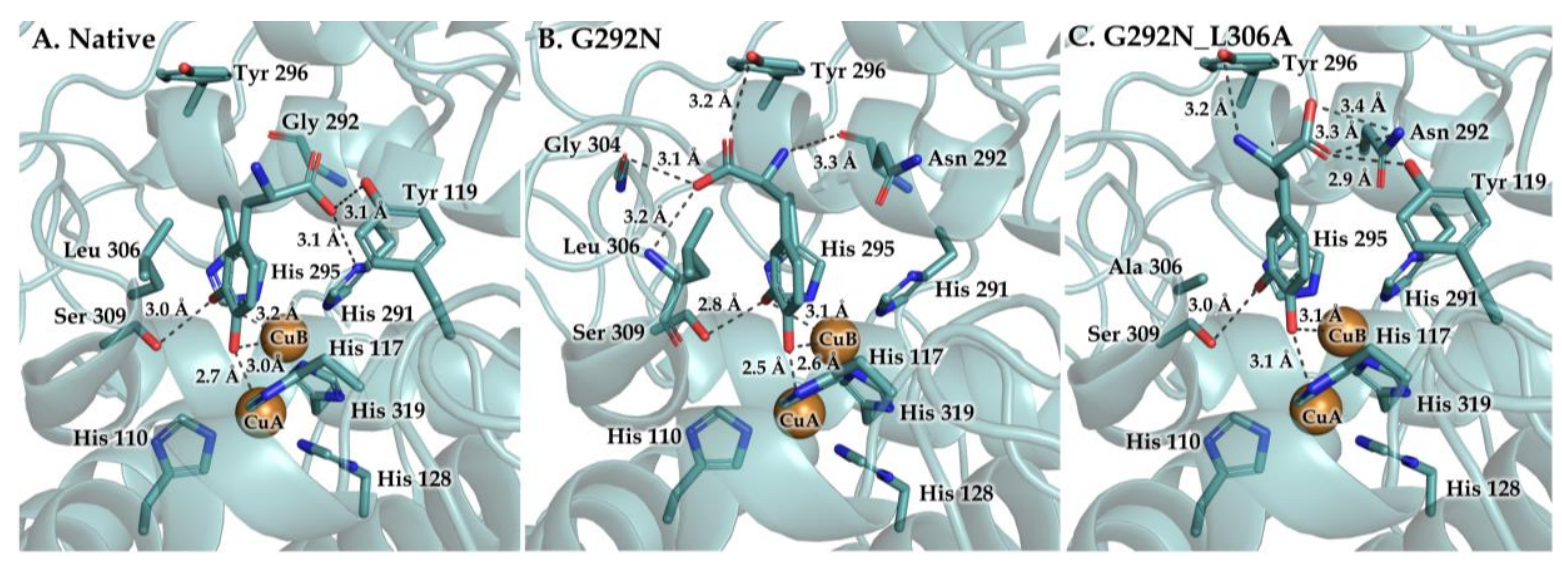

Figure 1. Docking poses of L-DOPA in (A) Native TtPPO, (B) variants G292N and variant G292N_L306A (C).

\section{Conclusions}

Previous studies concerning the structure-function relations of PPOs, with a special focus on the amino acids affecting the ratio of monophenolase/diphenolase activity, have revealed the implication of (a) the "gate" residue [18], positioned on top of CuA (in our case L306) and (B) the residue adjacent to the first $\mathrm{CuB}$ coordinating histidine residue ( $\mathrm{H}_{\mathrm{B} 1+1}$, in our case N292) [19]. Docking of L-DOPA in $T t P P O$ active site corroborates the involvement of these residues in the positioning of the substrate. These findings, combined with biochemical experiments, set the basis for structure-based design of TtPPO mutants with desired activity.

Author Contributions: Conceptualization, M.D.; Methodology and data analysis M.D., A.V. and G.D. Writingoriginal draft preparation, M.D., A.V., G.D,.; Writing - review and editing, M.D. and A.V.

Funding: This research was funded by EDBM103 Action "Support of researchers with emphasis on young researchers", NSRF 2014-2020.

Acknowledgments: Authors would like to thank Assoc Prof. Evangelos Topakas and Dr. Stratos Nikolaivits from National Technical University of Athens, School of Chemical Engineering for their continuous input, advice and support during this research.

Conflicts of Interest: The authors declare no conflict of interest.

\section{References}


1. Goldfeder, M.; Kanteev, M.; Isaschar-Ovdat, S.; Adir, N.; Fishman, A. Determination of tyrosinase substrate-binding modes reveals mechanistic differences between type-3 copper proteins. Nature Communications, 2014, 5. https://doi.org/10.1038/ncomms5505

2. Kim, H.; Yeon, Y. J.; Choi, Y. R.; Song, W.; Pack, S. P.; Choi, Y. S. A cold-adapted tyrosinase with an abnormally high monophenolase/diphenolase activity ratio originating from the marine archaeon Candidatus Nitrosopumilus koreensis. Biotechnol. Lett., 2016, 38(9), 1535-1542. https://doi.org/10.1007/s10529-016-2125-0

3. Lai, X.; Soler-Lopez, M. ; Wichers, H. J. ; \& Dijkstra, B. W. Large-Scale Recombinant Expression and Purification of Human Tyrosinase Suitable for Structural Studies. PLOS ONE, 2016, 11(8), e0161697. Retrieved from https://doi.org/10.1371/journal.pone.0161697

4. Li, Y.; Wang, Y.; Jiang, H.; Deng, J. Crystal structure of Manduca sexta prophenoloxidase provides insights into the mechanism of type 3 copper enzymes. PNAS USA, 2009, 106(40), 17002-17006. https://doi.org/10.1073/pnas.0906095106

5. Mauracher, S. G.; Molitor, C.; Michael, C.; Kragl, M.; Rizzi, A.; Rompel, A. High level protein-purification allows the unambiguous polypeptide determination of latent isoform PPO4 of mushroom tyrosinase Dedicated to Prof. Dr. H. Witzel on the occasion of his 90th birthday. Phytochemistry, 2014, 99, $14-25$. https://doi.org/10.1016/j.phytochem.2013.12.016

6. Kanteev, M.; Goldfeder, M.; Fishman, A. Structure-function correlations in tyrosinases. Protein Sci., 2015, 24(9), 1360-1369. https://doi.org/10.1002/pro.2734

7. Pretzler, M.; Rompel, A. What causes the different functionality in type-III-copper enzymes? A state of the art perspective. Inorganica Chim. Acta, 2018, 481, 25-31. https://doi.org/10.1016/j.ica.2017.04.041

8. Solano, F. Melanins: Skin Pigments and Much More-Types, Structural Models, Biological Functions, and Formation Routes. New J. Sci, 2014, 1-28. https://doi.org/10.1155/2014/498276

9. Gul, I. Polyphenol oxidase (PPO) based biosensors for detection of phenolic compounds: A Review. J. Appl. Biol. Biotechnol. 2017, 5(03), 72-85. https://doi.org/10.7324/JABB.2017.50313

10. Mukherjee, S.; Basak, B.; Bhunia, B.; Dey, A.; Mondal, B. Potential use of polyphenol oxidases (PPO) in the bioremediation of phenolic contaminants containing industrial wastewater. Rev. Environ. Sci. Biotechnol. 2013, 12, 61-73. https://doi.org/10.1007/s11157-012-9302-y

11. Panis F.; Rompel A. Identification of the amino acid position controlling the different enzymatic activities in walnut tyrosinase isoenzymes (jrPPO1 and jrPPO2). Sci. Rep. 2020, 10(1), 1-12. https://doi.org/10.1038/s41598-020-67415-6

12. Nikolaivits, E.; Dimarogona, M.; Karagiannaki, I.; Chalima, A.; Fishman, A.; Topakas, E. Versatile Fungal Polyphenol Oxidase with Chlorophenol Bioremediation Potential: Characterization and Protein Engineering. Appl. Environ. Microbiol. 2018, 84, e01628-18, /aem/84/23/e01628-18.atom. https://doi.org/10.1128/AEM.01628-18

13. Krieger, E.; Vriend, G. YASARA View - molecular graphics for all devices - from smartphones to workstations. Bioinforma. Oxf. Engl. 2014, 30, 2981-2982. https://doi.org/10.1093/bioinformatics/btu426

14. Feng, Z.; Chen, L.; Maddula, H.; Akcan, O.; Oughtred, R.; Berman, H.M.; Westbrook, J., Ligand Depot: a data warehouse for ligands bound to macromolecules. Bioinforma. Oxf. Engl. 2004, 20, 2153-2155. https://doi.org/10.1093/bioinformatics/bth214

15. Goodsell, D.S.; Morris, G.M.; Olson, A.J. Automated docking of flexible ligands: applications of AutoDock. JMR. 1996. 9, 1-5. https://doi.org/10.1002/(sici)1099-1352(199601)9:1<1::aid-jmr241>3.0.co;2-6

16. Morris, G.M.; Huey, R.; Lindstrom, W.; Sanner, M.F.; Belew, R.K.; Goodsell, D.S.; Olson, A.J. AutoDock4 and AutoDockTools4: Automated docking with selective receptor flexibility. J. Comput. Chem. 2009, 30, 2785-2791. https://doi.org/10.1002/jcc.21256

17. Trott, O.; Olson, A.J. AutoDock Vina: Improving the speed and accuracy of docking with a new scoring function, efficient optimization, and multithreading. J. Comput. Chem. 2009, 31(2), 455-461. https://doi.org/10.1002/jcc.21334

18. Penttinen, L.; Rutanen, C.; Jänis, J.; Rouvinen, J.; Hakulinen, N.; Unraveling substrate specificity and catalytic promiscuity of Aspergillus oryzae catechol oxidase, ChemBioChem. 2018, 19, 2348-2352. https://doi.org/10.1002/cbic.201800387

19. Solem, E.; Tuczek, F.; Decker, H. Tyrosinase versus catechol oxidase: One asparagine makes the difference. Angew. Chemie Int. Ed. 2016, 55, 2884-2888. https://doi.org/10.1002/anie.201508534. 
Publisher's Note: MDPI stays neutral with regard to jurisdictional claims in published maps and institutional affiliations.

(C) 2020 by the authors. Submitted for possible open access publication under the terms and conditions of the Creative Commons Attribution (CC BY) license (http://creativecommons.org/licenses/by/4.0/). 